\title{
A Logical Approach to Experience-Based Reasoning
}

\author{
Zhaohao Sun \\ Department of Business Studies \\ PNG University of Technology, Lae 411, PNG \\ $\&$ \\ School of Engineering and IT, Federation University Australia, Ballarat, Vic 3353, Australia \\ zhaohao.sun@pnguot.ac.pg; z.sun@federation.edu.au; zhaohao.sun@gmail.com
}

\begin{abstract}
Experience-based reasoning (EBR) is a paradigm used in almost every human activity as a part of human reasoning. However, EBR has not been seriously studied from a logical viewpoint. This article will attempt to fill this gap by providing a unified logical approach to EBR. More specifically, this article first examines EBR and inference rules. Then it proposes eight different rules of inference for EBR, which cover all possible EBRs from a logical viewpoint. These eight different rules of inference constitute the fundamentals for all EBR paradigms, and therefore will be the theoretical foundation for EBR. The proposed approach will facilitate research and development of EBR, human reasoning, and common sense reasoning.
\end{abstract}

Keywords: Experience-based reasoning, case-based reasoning, human reasoning, experience management.

\section{Introduction}

Experience has become even more important in the age of Internet and social media [1], because over $70 \%$ of online shopping is based on customer experience and experience based reasoning. For example, the authors recently bought a mobile phone based on the experience of their friend, who bought it online and used for one year and advised us that this brand of mobile phone is the excellent one from a viewpoint of performance and price. Experience-based Reasoning (EBR) is a reasoning paradigm using prior experiences to solve problems [2]. As well-known, experience is an important asset for a domain expert like a clinic doctor. However, how to formalize experience remains a significant issue, although 
customer experience analytics has drawn some attention in order to provide comprehensive and ever-evolving representation of the user experience [1]. Further, there is no fundamental research to investigate the logical or mathematical foundation of EBR.

Case-based reasoning (CBR) has sometimes been referred to as EBR [3]. Previous work on EBR in the context of CBR has investigated the use of modification operators and rules for routine case adaptation [4], and building of an experience factory and experience base in the software engineering domain [5]. One of the most important issues in EBR is to identify different types of knowledge and reasoning useful for different classes of case adaptation tasks [4]. However, CBR cannot cover all possibilities of EBR and should be considered a kind of EBR [6].

Human reasoning is an important topic for human intelligence. Johnson-Laird, Khemlani, \& Goodwin [7] addresses long-standing puzzle of how logic and probability fit together in human reasoning. However, their research only focuses on integrating probability with deductive reasoning, the latter can only be considered as a part of human reasoning, deductive reasoning. Human reasoning also includes non-deductive reasoning [8] such as abductive reasoning and reasoning with trick or deception [9]. Furthermore, human reasoning also includes invalid reasoning although a valid reasoning is one in which the conclusion is true in all cases in which the premises are true [7]. Therefore, how to formulize human reasoning is still a fundamental question.

This article attempts to fill this gap by providing a unified logical treatment of EBR as a part of human reasoning. More specifically, this article first examines EBR and reasoning and inference rules (or rules of inference). Then, it looks at eight different rules of inference of EBR, which cover all possible EBRs from a logical viewpoint. These eight different rules of inference constitute the fundamentals for all EBR paradigms, and therefore will be the theoretical foundation for EBR and human reasoning. The key idea behind it is that we classify EBR from a logical viewpoint and examine the correspondence between such a classification and real world problems using an inference rule with examples. We argue that the proposed approach in this article will facilitate the understanding of experience, EBR, their further development and their applications to experience management (EM) and human reasoning.

It should be noted that although our approach might be of significance in areas such as fuzzy reasoning [10], common sense reasoning [11], human reasoning [7], natural reasoning, 
experience engineering and multiagent systems [3], we will not attempt to discuss its applications in these or related areas in the present article.

The rest of this article is organized as follows: Section 2 examines experience based reasoning. Section 3 briefly looks at reasoning and inference rules. Section 4 explores inference rules for experience based reasoning with applications. The final sections discuss the related work and end this article with some concluding remarks and future work.

\section{Experience-based Reasoning}

This section will examine experience-based reasoning (EBR) in a general setting. It argues that EBR as a kind of human reasoning is important enough for us to formalize it. In order to do so, we first illustrate EBR with examples.

Let $X$ and $Y$ be two classmates. They attended the same lectures during their university study. Therefore, they possess the same knowledge. However, they use different methods to learn knowledge and language, and use tools available to themselves so that they have different examination results. In fact, they also have a different future.

This is a typical example. It implies that knowledge, language, and inference rules are only tools for us to use to realize our goal. How to learn language and knowledge belongs to the category of experience; that is, how to select tools is a kind of EBR. From a logical viewpoint, how to learn language and knowledge, how to use inference rules, and which tools should be selected for use can be considered as meta-knowledge, meta-language, and meta-inference rules respectively [3]. Therefore meta-knowledge, meta-language, and meta-inference rules are fundamentals for EBR, although they are still open problems.

Let us look at another example [6]:

Peter Hagen is a famous Professor of Business at the University of Trickland ${ }^{1}$. He has organized and chaired many international conferences and visited many different countries for academic travels. He teaches his student logistics using modus ponens and modus tollens [12], while he explains some social phenomena using abductive reasoning [13]. When he participates in a business negotiation with his competition, he likes to use modus ponens with trick and modus tollens with trick [6]. He also likes to conduct some investment, in which he

\footnotetext{
${ }^{1}$ This is a virtual name.
} 
likes to use inverse modus ponens and inverse modus tollens. In other matters, he sometimes uses inverse modus ponens with trick and inverse modus tollens with trick.

From this example, we can see that

1. any human professional activities usually involve the application of many reasoning paradigms such as abduction and deduction. The fundamental question from here is: how many reasoning paradigms we have for EBR and human reasoning?

2. which tools (including inference rules and reasoning paradigms) should be selected for use is also a kind of EBR. Moreover, selecting the most appropriate tool at a right place, at a right time to solve a real-world problem in an optimal way is a kind of EBR [14].

In what follows, we focus on the exploration of inference rules as a part of reasoning paradigms for EBR at a fundamental level.

\section{Reasoning and Inference Rules}

Reasoning is the act of using reason to derive a conclusion from certain premises using a given methodology ${ }^{2}$. The most important reasoning form, or so-called neat or clean and flawless form that we have ever used, is deductive reasoning [7] [15]. Many other reasoning paradigms can be considered as an extension or revision of deductive reasoning. For example, fuzzy reasoning is an extension of deductive reasoning [3]. Default reasoning is the revision of deductive reasoning taking into account discovery of processing exceptions.

Reasoning is a central concept in knowledge based systems and Artificial Intelligence (AI) [16], where there are a few dozen reasoning paradigms that have been studied such as non-monotonic reasoning, default reasoning, fuzzy reasoning, probabilistic reasoning, CBR, EBR and human reasoning, to name a few.

As well known, reasoning can be used for problem solving [7]. However, one reasoning paradigm, in particular "faultless deduction" [15] is not enough to solve all real world problems. It is better to combine several different reasoning paradigms to solve one real-world problem. For example, deductive reasoning and abductive reasoning can be integrated to solve a class of real problems such as diagnosis and treatment encountered in

\footnotetext{
${ }^{2}$ http://en.wikipedia.org/wiki/Reasoning
} 
clinical practice [9]. More specifically, abductive reasoning is used to generate an idea or explanation for solving the problem like diagnosis, and then deductive reasoning is used to really solve the problem like treatment in the clinic. Sometimes, experience based reasoning (EBR) and similarity-based reasoning is necessary to solve a problem. This is the reason why abductive CBR and deductive CBR are proposed and their integration are emphasized in order to solve some problems [9]. Therefore, one reasoning paradigm can be only used through EBR or human reasoning to solve a class of real world problems. This is the reason why researchers propose abduction, induction, and other reasoning paradigms [16].

In mathematical logic, reasoning can be considered as a process of manipulating logical language based on inference rules to infer a new conclusion in logical language [17]. The reasoning process for solving a real world problem makes up a reasoning chain [15]. In this reasoning chain consisting of a number of nodes, the reasoning from one node to another is based on one or more inference rules. An inference rule is a scheme for constructing valid inferences [18]. These schemes establish syntactic relations between a set of formulas called premises and an assertion called a conclusion. These syntactic relations are used in the process of inference, whereby new true assertions are arrived at from other already known ones. Therefore, inference rules are the basis for any reasoning paradigm in EBR and human reasoning.

There are more than 30 inference rules $^{3}$ for natural deduction, which constitutes a formalism that models deductive reasoning involved in mathematical proofs, proposed by Gerhard Gentzen in 1934 [19, p. 4], who attempted to find a more natural approach to actual reasoning in mathematical proofs.

Furthermore, there is a hierarchy among these existing reasoning paradigms. For example, similarity based reasoning (SBR) is not at the first level in the hierarchy of reasoning paradigms. We can consider SBR as a combination of deduction and similarity, we can also consider SBR as a combination of abduction and similarity [6]. From a viewpoint of Boolean algebra, inference rules such as modus ponens and modus tollens are at the atomic level, because each of them constitutes an edge linking two different nodes in the reasoning chain, and they cannot be further broken down like the above mentioned SBR. How many inference rules at the atomic level is not only an interesting issue but also a fundamental question for EBR and human reasoning. In what follows, we will address this issue, whereas Gentzen

\footnotetext{
${ }^{3}$ In fact, Gentzen proposed 28 inference rules for natural deduction. However, there are still 26 well-known inference rules in propositional logic [18].
} 
looked at inference rules for deductive reasoning.

\section{Inference Rules for Experience Based Reasoning}

As we know, one of the most important principles of human reasoning including EBR is "divide and conquer"; that is, we first divide a real world problem so simply that we can conquer the divided problem using existing reasoning or methods [6]. Based on this principle, we will classify EBR using inference rules from a logical viewpoint and examine the correspondence between such a classification (inference rules for EBR) and real world problems using an inference rule. In what follows, we outline four existing inference rules and propose four new inference rules for EBR with examples. We will explain the informal sense of each inference rule and thus try to show how the inference rules reflect EBR. These eight different inference rules, four of which having not drawn plenty of attention in either logic or computer science, constitute the fundamentals for all kinds of EBR paradigms at the atomic level taking into the structure of Boolean algebra [12].

\subsection{Modus Ponens and Modus Tollens}

The most common inference rules are the modus ponens (MP) and modus tollens $(M T)$, which have played an important role in mathematics and computer science [16] [10]. These two reasoning paradigms underlie almost every field of science, engineering, technology, business and management [7].

$M P$ has the following general form:

$$
\frac{P}{P \rightarrow Q}
$$

where $P, Q$ represent any (compound) propositions whatsoever such as "Beijing is a small city". More specifically, MP (1) means that if $P$ is true, and $P \rightarrow Q$ is true, then the conclusion $Q$ is also true. Strictly speaking, (1) can be considered as a part of proof theory, while, the explanation of MP (1) belongs to model theory, which determines the validity of inferences [7].

MP is the important rule of inference in mathematics, because it is the foundation of forward chaining (together with hypothetical syllogism [12, p. 33] in problem solving. The term modus ponens is Latin meaning "method of affirming", since the conclusion is an affirmation [12]. From an EBR viewpoint, Form (1), is a formalized summary and abstraction of experience encountered by many people in the past. For example, 
Example 1. Modus ponens. We have the knowledge in the knowledge base:

1. If Socrates is human, then Socrates is mortal

2. Socrates is human

What we wish is to prove "Socrates is mortal". In order to do so, let

- $P \rightarrow Q$ : If Socrates is human, then Socrates is mortal,

- $P$ : Socrates is human

- $Q$ : Socrates is mortal

Therefore, we have $Q$ : Socrates is mortal using modus ponens (1). Error! Reference source not found.In what follows, we turn to modus tollens (MT). The general form of MT is as follows:

$$
\begin{gathered}
\neg Q \\
P \rightarrow Q \\
\therefore \neg P
\end{gathered}
$$

where $P, Q$ represent compound propositions. More specifically, MT (2) means that if $Q$ is false, and $P \rightarrow Q$ is true, then the conclusion $P$ is also false.

MT is also one of the most important rules of inference in mathematics, because it is the foundation of backward chaining (together with hypothetical syllogism [12, p. 33] in problem solving.

The term modus tollens is Latin meaning "the method of denying", since the conclusion is a denial.

Example 2. Modus tollens. We have the knowledge in the knowledge base:

1. If Peter is healthy, then Peter will fly to Beijing

2. Peter will not fly to Beijing

What we wish is to prove "Peter is not healthy". In order to do so, let

- $P \rightarrow Q$ : If Peter is healthy, then Peter will fly to Beijing,

- $P$ : Peter is healthy

- $Q$ : Peter will fly to Beijing

Therefore, we have $\neg P$ : Peter is not healthy, based on modus tollens (2), and the knowledge in the knowledge base.

Modus ponens (MP) and modus tollens (MT) are two basic rules of inference related to conditional statements in mathematical logic. In fact, they have also played an important role in problem solving in mathematics and modes of argumentation as well as in AI. Further, in mathematical reasoning, MT is used almost as often as MP [12]. Based on the above 
discussion, MP and MT are also two basic inference rules for EBR. From a theoretical viewpoint, many knowledge based systems (KBS) in particular rule-based expert systems [11] are also based on these two inference rules, therefore, KBS are also a form of EBR systems.

It should be noted that MP and MT belong to rules of deduction, which are reasoning paradigms in mathematical logic, mathematics and AI. The reasoning (or argument) using them can be considered as valid; that is, no matter what particular statements are substituted for the statement variables in its premises, if the resulting premises are all true, then the conclusion is also true $[12,7]$.

\subsection{Abduction}

Abduction plays a fundamental role in problem solving [20] [21]. In particular, abduction seems to be a basic reasoning component in activities such as system explanation [8] and diagnosis [21] as well as system analysis [6]. Abduction is becoming an increasingly popular term in many fields of computer science such as system diagnosis, planning, natural language processing, and logic programming [22] [21]. Kindler et al. applied abduction and deduction to the laboratory medicine problem solving process [23]. CBR has played an important role in explanatory or abductive reasoning tasks like diagnosis and explanation, one of which is case-based explanation [8] and abductive CBR [9]. In most AI views, explanations are treated as deductive proofs [9]. Abductive reasoning systems build their proofs by non-deductive methods, and additional assumptions may be required for those proofs to apply [8]. However, their view is fundamentally the same in that if the abductive assumptions were shown to be true, the resulting explanation would be considered a deductive proof [9]. Abduction has also been drawn increasing attention in philosophy and cognitive science. For example, Magnani integrates philosophical, cognitive and computational issues on abduction, examines some cases of reasoning in science and medicine, shows the connections between abduction, induction, and deduction, and argues that abduction is a logic of scientific discovery [24].

The general model of abduction as a rule of inference is as follows [22]:

$Q$
$\frac{P \rightarrow Q}{\therefore P}$

where $P$ and $Q$ represent any (compound) propositions in a general setting ${ }^{4}$. In clinical

${ }^{4}$ From now on, we do not mention this fact about $P$ and $Q$ any more when we introduce a new rule of inference. 
diagnosis, $P \rightarrow Q$ is a form of general relation: disease $\rightarrow$ symptom [9]

Abduction (3) is also a formalized summary and abstraction of experience encountered by many people in the past.

Example 3. Abduction (borrowed from [24, p. 21] and heavily revised). It is a very simple example dealing with diagnostic reasoning [9]. We will begin with the situation as it might be described in English: the knowledge (sentences) in the knowledge base includes:

1. If a patient is affected by a pneumonia, his/her level of white blood cells is increased,

2. John's level of white blood cells is increased.

What we wish is to prove "John is affected by pneumonia" using abduction (3). We first represent these facts in a first-order logic, and then show the proof as a sequence of applying abduction (3). To this end, let $P(x): x$ is affected by a pneumonia, $Q(x): x$ 's level of white blood cells is increased, $P(J o h n): J o h n$ is affected by a pneumonia, Q(John): John's level of white blood cells is increased. Then the above example can be formalized as:

1': $\forall x(P(x) \rightarrow Q(x))$

2': Q(John)

We use the substitution $\{x / \mathrm{John}\}$ (for detail see [11]) or an inference rule (elimination of quantifier) [17], and infer:

3': $P($ John $) \rightarrow Q(J o h n)$

From (2') and (3'), and abduction (3), we have: $P(J o h n)$; that is, John is affected by a pneumonia 5 .

The above example of diagnostic reasoning is an excellent way to introduce abduction [24, p. 18]. In fact, abduction goes back more than a hundred years. At that time, the American philosopher Charles Sanders Peirce defined "Abduction" as inference that involves the generation and evaluation of an explanatory hypothesis [24].

From an epistemological viewpoint [24, p. 25], there are two main meanings of the word abduction: (1) abduction is only to generate plausible hypotheses (selective or creative) and

${ }^{5}$ It should be noted that the above argument is invalid in the context of traditional mathematical logic. The fallacy underlying this invalid argument form is called the converse error [12] (p. 36). However, it is possible for a valid argument to have a false conclusion, and for an invalid argument to have a true conclusion. The latter case often happens in EBR. We will see such cases; that is, an invalid argument with a true conclusion, in the basic rules of inference for EBR. 
(2) abduction can be considered as an inference to the best explanation, which also evaluates hypotheses. The latter is the meaning of abduction accepted in this article, while the first meaning of abduction was accepted by Magnani.

The study of abductive inference was slow to develop, although it mentioned by Aristotle more than two thousand years ago. The reason for this might be that abduction lacks certain logical properties such as completeness, soundness, satisfaction, consistency and decidability. Furthermore, logicians have concentrated on deductive inference or systems [17]. Abduction is the term currently used in AI for generation of explanations for a set of events from a given domain theoryError! Reference source not found. More specifically, abduction is the process of inferring certain facts and/or laws and hypotheses that render some sentences plausible, that explain or discover some (eventually new) phenomenon or observation; it is the process of reasoning in which explanatory hypotheses are formed and evaluated [24, $\mathrm{p}$. $18]$.

From a logical viewpoint, abduction is an unsound reasoning [22]. However, it has similar properties to those of other non-monotonic logics in the AI literature. For example, it shares declarative and computational properties with other forms of nonmonotonic reasoning [9].

Minsky doubts that pure deductive logic plays much of a role in ordinary thinking [15, p. 189], although it can help us find the most essential steps, once we find a way to solve a certain problem. However, he has not examined how to find a way at all. We believe that abduction can serve to do so, at least abduction is an important complement to deduction, because abduction is a very useful nonmonotonic reasoning, in particular for reasoning towards explanation in (system) diagnosis [13] and analysis in problem solving, and therefore an important form of EBR.

\subsection{Reasoning with Trick}

The reasoning with trick was introduced by Sun and Weber [26], and aims at examining logic with trick and reasoning with trick ${ }^{6}$. This section will review this kind of reasoning and propose reasoning with trick as a kind of EBR and human reasoning.

\subsubsection{Introduction}

Turing proposed the imitation game, known as the Turing test, in 1950 [27]. If a computer program could pass the Turing test, then it could be said to be intelligent [26]. No doubt, the

\footnotetext{
${ }^{6}$ We use the term "trick" to cover several reasoning approaches including deception and cheat.
} 
Turing test and Turing's seminal article 'Computing machinery and intelligence' [27] led to the serious research and development of AI such as natural language translation, game playing, automatic theorem proving, and machine learning in the following 65 years [22] [11]. However, we have to admit a fact that we have not yet studied the tricks involved in the Turing test seriously [28], although a trick is an important component of human intelligence. Many have been yet unaware of the intelligence with trick involved in the Turing test, although a few such as [29] and [28] have already asserted that when someday a computer program does pass the Turing test, it will use many tricks, for the simple reason that people already use them often.

Turing [27] also hinted that it was impossible to make a Turing system successful without rigorous study on intelligence with trick and ingenious use of intelligence with trick in it. It seems very likely that the result of neglecting this hint is that no computer programs have yet passed the Turing test (http://www.loebner.net/Prizef/loebner-prize.html, retrieved on 18 Jan 2016). The Internet and social media make the passing of Turing Test more complex, because it is difficult to differentiate an intelligent agent from a real physical person. It is also difficult for us to know we should learn from intelligent agents or verse vice.

Intelligence with trick is the appearance of such negative reasoning "to cheat and trick" included in human reasoning [26]. Its reasoning, different from monotonic reasoning and non-monotonic reasoning such as default reasoning, autoepistemic reasoning and circumscription [22], reflects such human reasoning with trick and cheat as "make a feint to the east and attack in the west" [26].

\subsubsection{Where is a Trick from?}

There are many different types of tricks ${ }^{7}$ in human reasoning, in particular, in wars, in politics or in business. One of the well-known military books written by the Chinese ancient military scientist Sun Tzu over 2000 years ago is "The Art of War" (the current version can read [30]. In that book there are many different major tricks/deceptions (rather than tactics) which are appropriate to certain military occasions. Furthermore, virtually, everybody has at least one experience in deceiving or cheating another person or being deceived or cheated by someone [26]. Therefore we can assert that there is no development of human intelligence without tricks, in particular, it is true for the development of intelligence of an individual.

\footnotetext{
${ }^{7}$ Note that the main goal of performing trick or deception is to get interest advantage.
} 
Then, where is a trick from? We think that in a most general sense, if there is a difference between two agents whether in possessing knowledge or in thinking methods or reasoning strategies, then there can be a trick between them [14]. Therefore, there are three different possibilities to lead to a trick. One possibility is that a trick comes from common background knowledge but different reasoning rules of the two agents (for convenience, $C$, and $D$ ). For example, both agents have a common knowledge: $P$ and If $P$ then $Q$. If one (for example, $C$ ) uses modus ponens, while D doesn't, then a trick may occur. Another possibility is that a trick comes from different background knowledge, although using common inference rule(s), i.e. $C$ has knowledge $\mathrm{A}^{*}$ and at the same time $D$ has different knowledge $\mathrm{A}$, whereas both share the same inference rules, e.g. $P, P \rightarrow Q \Rightarrow Q$. Thus the disjoint sum $A^{*}+A \neq \emptyset$ leads to a trick, because $C$ thinks $D$ would obtain the same conclusion, but he won't. The third possibility is that a trick comes from both different knowledge and different inference rules between two agents [6]. In the following we further develop the above-mentioned ideas from a logic viewpoint.

\subsubsection{Modus Ponens with Trick}

We do not pursue logic with trick and reasoning with trick any further in this article, but we turn to basic inference rules with tricks. The key idea is that the trick will perhaps happen whenever there are differences between two individual's knowledge, experience and reasoning.

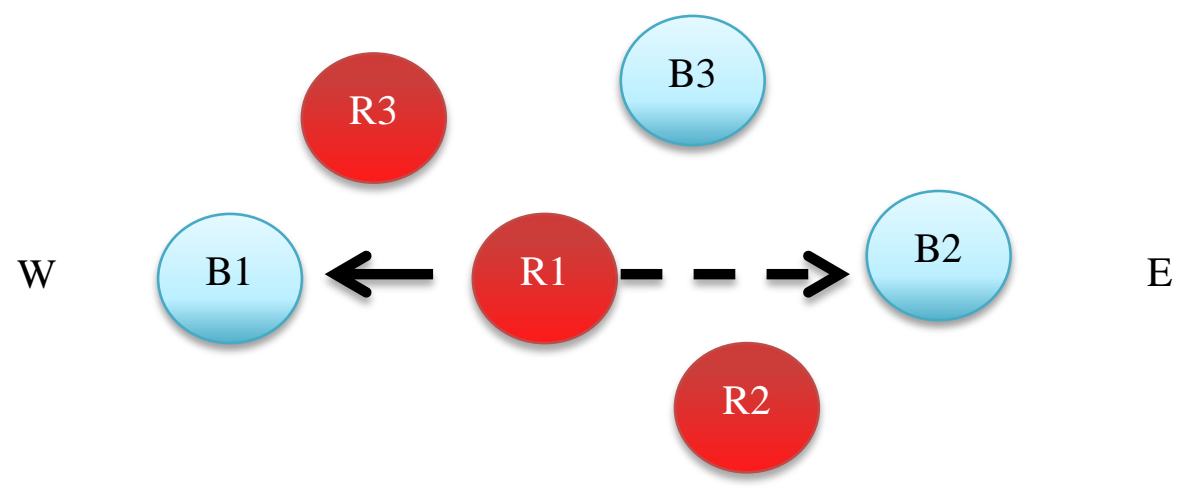

Fig 1. A trick-driven military action

Example 4. Modus ponens with trick. There was a military situation as follows (also see Fig 1): Units of the blue army (B) and units of the red army (R) confronted each other. They had a common wish to defeat the other side as soon as possible. The commander of the blue army believed firmly that if it was raining, then the red army would carry out an attack eastwards. With the start of rain, based on the above analysis, he commanded his units to 
defend themselves against the attack from the east. However, the commander of the red army used the deception-based tactic of "make a feint to the east and attack in the west" and carried out a surprise attack westwards and in the end conquered the blue army.

One concrete story for this example is the following example:

Example 5. A real example of reasoning with trick [31]. In World War I the German headquarters used reasoning with trick in preparation of the attack on France. At that time, Belgium, which is located north of France, was a neutral state. Although the French supreme command speculated that the German attack would try to outflank their eastern fortress line, they thought that this attack wouldn't touch the north of Belgium, because of concern of the British reaction. The German headquarters didn't reason in this way. In spite of British fears they overran and occupied the whole of Belgium and attacked France along almost its entire northern border.

From these examples we can see that the reasoning with trick usually runs counter to normal thinking or reasoning in order to obtain a certain advantage. We can generalize this idea and obtain the following rule of inference with trick, which is called modus ponens with trick:

$$
\begin{gathered}
P \\
P \rightarrow Q \\
\therefore \quad \neg Q
\end{gathered}
$$

It should be noted that the reasoning based on modus ponens with trick is invalid in the traditional framework of mathematical logic and mathematics [12, p. 36]. However, it is useful for war, business and negotiation, therefore, we consider it as a basic rule of inference for EBR.

Multiagent systems (MAS) are a moving research field in AI [32]. Cooperation, coordination, communication and negotiation play an important role in the system intelligence of MAS [3]. However, these are the basic component for a community so that social intelligence becomes an interesting topic in MAS. From a viewpoint of the human reasoning, we can divide the social behavior into two categories. The first includes autonomy and trust, while the latter includes deception and lies. Therefore, the investigation into deception or trick among intelligent agents in MAS [33] and deceptive reasoning in MAS [3] can help not only improving better understanding of human reasoning and EBR, but also improving intelligent EBR systems and MAS. 


\subsection{Modus Tollens with Trick}

We have discussed modus tollens in Section 4.1. Now we examine its "dual" form, named modus tollens with trick. Its general form is

$$
\begin{gathered}
\neg Q \\
P \rightarrow Q \\
\hline \therefore P
\end{gathered}
$$

Theoretically speaking, it is also a variant of modus ponens with trick, because in the traditional mathematical logic, we have $\neg Q, P \rightarrow Q \Rightarrow \neg P$ and $\neg \neg P \Leftrightarrow P$. However, in non-traditional logic, for example, in fuzzy logic [10], $P \rightarrow Q \Leftrightarrow \neg Q \rightarrow \neg P$ and $\neg \neg P \Leftrightarrow P$ are normally invalid. In particular in EBR, they both can be invalid, therefore modus tollens with trick is still meaningful in order to examine the basic rule of inference in EBR. In what follows, we will give an example to verify the usefulness of modus tollens with trick.

In some cases, if $Q$ is false, then we do not evaluate if $P \rightarrow Q$ is true, and conclude that $P$ is true. This is the essence of modus tollens with trick from a logical viewpoint. This consideration is similar to the logical operator, "And Also", which is the same as the And operator, except it performs short circuit evaluation [34, p. 230]; that is, if $P$ is false, then $P$ AndAlso $Q$ is false. Therefore the introduction of modus tollens with trick is also a new understanding of traditional logical operators.

Example 6. Modus tollens with trick. We have the knowledge in the knowledge base:

1. If Chris has an MBA Degree from Harvard University, then Chris is a millionaire

2. Chris is not a millionaire

What we wish is to prove "Chris has an MBA Degree from Harvard University". In order to do so, let

- $P \rightarrow Q$ : If Chris has an MBA Degree from Harvard University, then Chris is a millionaire

- $P$ : Chris has an MBA degree from Harvard University

- $Q$ : Chris is a millionaire

Therefore, we have $P$ : Chris has an MBA degree from Harvard University, based on modus tollens with trick (5) and the knowledge in the knowledge base (note that $\neg Q$ : Chris is not a millionaire).

This example shows that belief and knowledge are different from what we can get based on the traditional inference rules in mathematical logic and mathematics. In other words, mathematics and mathematical logic can only formalize some problems in our 
knowledgeable world, in particular some kinds of EBR. Further, in modus tollens with trick (5), $P \rightarrow Q$ can be considered as common sense or common experience encountered in the past. However, experience usually has an exception, so that not everyone with a Masters Degree from Harvard University has a big salary in his late career. The modus tollens with trick (5) as a rule of inference reveals this kind of exception.

\subsection{Abduction with Trick}

As discussed in Section 5.2, abduction is an important reasoning paradigm in EBR. Its "dual" form is abduction with trick, which is also the summary and abstraction of a kind of EBRs. The general form of abduction with trick, as a basic rule of inference, is as follows:

$$
\frac{P \rightarrow Q}{\therefore \neg P}
$$

The difference between abduction with trick and abduction is "with trick". This is because the reasoning performer tries to use tricks such as "make a feint to the east and attack in the west"; that is, he gets $\neg P$ rather than $\mathrm{P}$ in the abduction as a rule of inference. This also verifies that difference is the necessary condition for performing tricks or deceptions (see Section 5.3).

Example 7. Abduction with trick. We have the knowledge in the knowledge base:

1. If the quarter profit is increasing, then Klaus invests the Project on BAI (Big data intelligence),

2. Klaus invests the Project on BAI.

What we wish is to prove "the quarter profit is not increasing". To this end, let

- $P \rightarrow Q$ : If the quarter profit is increasing, then Klaus invests the Project on BAI,

- $P$ : The quarter profit is increasing,

- $Q$ : Klaus invests the Project on BAI.

Then we use the knowledge in the knowledge base to perform abduction with trick and obtain $\neg P$; that is, the quarter profit is not increasing.

This is a usual case in business decision making. In fact, this is a kind of explanation for why Klaus invests the Project BAT. The decision maker considers that "If the quarter profit is increasing, then Klaus invests the Project on BAI'. However, Klaus decides to invest the project on BAI, although, at the end, "the quarter profit is not increasing". Therefore this is also a kind of EBR. This is the reason why we consider it as a basic rule of inference in EBR. 


\subsection{Inverse Modus Ponens}

Inverse modus ponens is also a rule of inference in EBR. The general form of inverse modus ponens is as follows:

$$
\frac{P \rightarrow Q}{\therefore \neg Q}
$$

The "inverse" in the definition is motivated by the fact that the "inverse" is defined in mathematical logic: "if $\neg p$ then $\neg q$ ", provided that if $p$ then $q$ is given [12]. Based on this definition, the inverse of $P \rightarrow Q$ is $\neg P \rightarrow \neg Q$, and then from $\neg P, \quad \neg P \rightarrow \neg Q$ we have $\neg Q$ using modus ponens. Because $P \rightarrow Q$ and $\neg P \rightarrow \neg Q$ are not logically equivalent, the argument based on (7) is not valid in mathematical logic and mathematics. However, it is also a basic rule of inference in EBR.

Example 8. Inverse modus ponens: We have the knowledge in the knowledge base:

1. If Monica has money, then Monica will fly to Beijing for a visit

2. Monica has not money

What we wish is to prove "Monica will not fly to Beijing for a visit". In order to do so, let

- $P \rightarrow Q$ : If Monica has money, then Monica will fly to Beijing for a visit

- $P$ : Monica has money

- $Q$ : Monica will fly to Beijing for a visit.

Therefore, we have $\neg Q$ : Monica will not fly to Beijing for a visit, based on inverse modus ponens, and the knowledge in the knowledge base (note that $\neg P$ : Monica has not money).

As just mentioned, the argument using inverse modus ponens is not valid. However, the case mentioned in the example often happens in everyday life. For example, in Australian Universities the following EBR based on inverse modus ponens holds:

Example 9. We have the knowledge: If the number of new students is increasing, then Ausdy (which is an artificial name) University employs a lecturer in Information Technology. Now the Ausdy University faces the fact that the number of new students is declining. Therefore, Audy University does not employ a lecturer in Information Technology anymore.

From this example we can see that invalid reasoning is allowable in EBR, if the consequence of reasoning is meaningful for decision making for a real world problem. In fact, if the decision-maker uses reasoning with trick, then he basically does not care whether the 
reasoning or argument is valid (which is a mathematical concept). What he emphasizes is if the reasoning conclusion is true, right or useful.

EBR based on inverse modus ponens is a kind of common sense reasoning, because there are many cases that follow inverse modus ponens. For example, if Peter has enough money, then Peter will buy the company Facebook. Now Peter does not have sufficient money, then we can conclude that Peter does not buy the company Facebook.

\subsection{Inverse modus ponens with trick}

The final inference rule for EBR is inverse modus ponens with trick. Its general form is as follows:

$$
\frac{P \rightarrow Q}{\therefore Q}
$$

The difference between inverse modus ponens with trick (8) and inverse modus ponens (7) is again "with trick", this is because the reasoning performer tries to use the trick of "make a feint to the east and attack in the west"; that is, he gets $Q$ rather than $\neg Q$ in the inverse modus ponens. This also verifies that difference is the necessary condition for performing trick or deception as mentioned in Section 5.3.

Example 10. Inverse modus ponens with trick. We have the knowledge in the knowledge base:

1. If the quarter profit is increasing, then Klaus invests the Project IMPT,

2. The quarter profit is not increasing.

What we wish is to prove "Klaus does invest the Project IMPT". In order to do so, let

- $P \rightarrow Q$ : If the quarter profit is increasing, then Klaus invests the Project IMPT,

- $P$ : The quarter profit is increasing,

- $Q$ : Klaus invests the Project IMPT.

Therefore, we have $Q$ : Klaus invests the Project IMPT using the above inverse modus ponens th trick and the knowledge in the knowledge base (note that $\neg P$ : the quarter profit is not increasing).

This is also a usual case in business decision making. Although the decision maker considers that "If the quarter profit is increasing, then Klaus invests the Project IMPT", at the end we have "The quarter profit has not increased". In this case, Klaus still has to invest the Project IMPT, based on a long run interest. The key idea behind this is that the decision must change with the changing situation. It is often the case for a strategic leader like CEO in a 
global company to apply above-mentioned inverse modus ponens with trick to make decision, in particular in the case of change of markets and developing new products.

\subsection{Review of Inference Rules for EBR}

Table 1 summarizes the examined eight basic inference rules with respect to EBR. It should be noted that some general forms in the table such as inverse modus

Table 1: Experience-Based Reasoning: Rules of Inference

\begin{tabular}{|c|c|c|c|c|c|c|c|}
\hline $\begin{array}{l}\text { monus } \\
\text { ponens }\end{array}$ & $\begin{array}{l}\text { monus } \\
\text { ponens } \\
\text { with trick }\end{array}$ & $\begin{array}{l}\text { inverse } \\
\text { monus } \\
\text { ponens } \\
\text { with trick }\end{array}$ & $\begin{array}{l}\text { inverse } \\
\text { monus } \\
\text { ponens }\end{array}$ & $\begin{array}{l}\text { modus } \\
\text { tollens }\end{array}$ & $\begin{array}{l}\text { modus } \\
\text { tollens with } \\
\text { trick }\end{array}$ & abduction & $\begin{array}{l}\text { abduction } \\
\text { with trick }\end{array}$ \\
\hline$P$ & $P$ & $\neg P$ & $\neg P$ & $\neg Q$ & $\neg Q$ & $Q$ & $Q$ \\
\hline$P \rightarrow Q$ & $P \rightarrow Q$ & $P \rightarrow Q$ & $P \rightarrow Q$ & $P \rightarrow Q$ & $P \rightarrow Q$ & $P \rightarrow Q$ & $P \rightarrow Q$ \\
\hline$\therefore Q$ & $\therefore \neg Q$ & $\therefore Q$ & $\therefore \neg Q$ & $\therefore \neg P$ & $\therefore P$ & $\therefore P$ & $\overline{\therefore \neg P}$ \\
\hline
\end{tabular}

ponens have received attention from some researchers [12, p. 36]. However, the researchers consider this inference rule as the source of fallacies in the reasoning, while we argue that they are all basic inference rules for EBR.

The key idea behind this view is that real world problems should be first classified and then verified if methodologies can be used to conquer the subproblems. Each of the subproblems can be investigated by a reasoning paradigm. A reasoning paradigm based on one inference rule has both theoretical significance and practical significance.

As we know, in logic, a hypothesis and conclusion are not required to have related subject matters $[12$, p. 26]. This assumption makes mathematical logic able to process symbolic reasoning. However, in EBR, a hypothesis and conclusion should have some related subject matter, therefore, we can claim that EBR is a reasoning towards practice and serves practical business and decision-making.

Based on the above investigation, we can see that CBR [35] [3] is a concrete realization of EBR. The research and development of CBR has provided us with some useful methodologies such as case base building, case reuse, case adaptation, case revision and case retention [36]. All these can be used for the EBR paradigm corresponding to each of the above proposed inference rules and become a new research branch of EBR. For example, abduction leads to abductive CBR [9]. Therefore, we can illustrate this consideration as relationship between EBR and CBR with Fig. 2, in which there are three levels (in fact, 
human reasoning should be at higher level than EBR, which is not illustrated in Fig. 2). Level 1 is EBR. Level 2 is EBR driven CBR. Level 3 consists of eight different CBR-based reasoning paradigms based on each of above proposed inference rules of EBR. The arrow $A \rightarrow \mathrm{B}$ means that $B$ is a part of $A$. for example, $\mathrm{CBR}$ is a part of EBR, while deductive CBR and abductive CBR [9] are parts of CBR, ..., so does Abductive CBR with trick. Therefore, this consideration through Fig 2 reveals the relationship between EBR and CBR and further provides CBR with 8 different CBR reasoning paradigms.

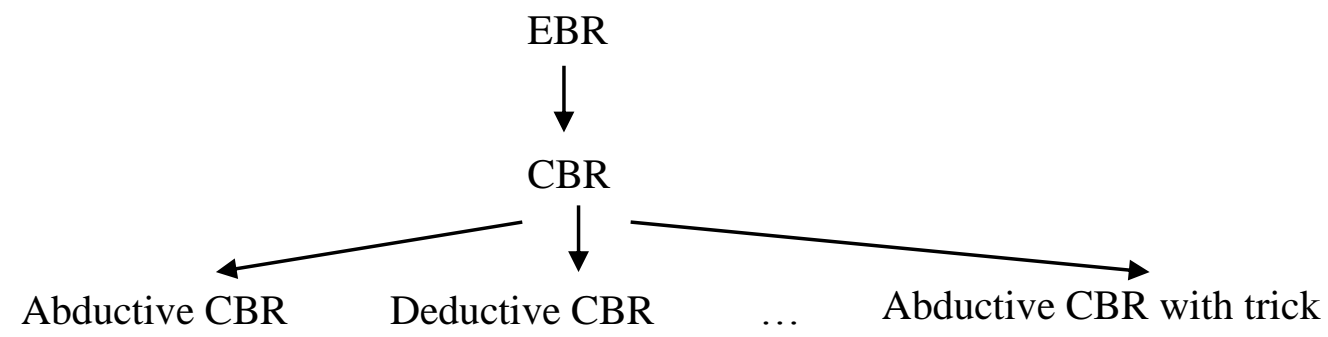

Fig 2. EBR and CBR

It should be noted that inductive reasoning (induction) is an important kind of reasoning paradigm in EBR. However, induction is a not an atomic reasoning like modus ponens, because it can be integrated into each of the above proposed and discussed reasoning paradigms for EBR. We would like to investigate it and its integration in a separated article.

\section{$5 \quad$ Related Work and Discussions}

As well-known, experience is a strategic asset for individuals in many fields such as clinic practice, on the one side. In particular, in Chinese medicine practice, an elder experienced doctor is more trustworthy. However, experience can kill people, on the other side. A number of generals were defeated in the battles because they made decisions and commands based on their experiences. A great number of successful businessmen lost their business because of experience as their tools for decision making. Therefore, experience always confuses us so that we do not know if we use experience as the tools for our reasoning for problem solving. However, these considerations are at too high or too general level. In order to make experience useful for us and mitigate its weakness, we have to go into lower level of the experience. This is the reason why we explore the reasoning paradigms of EBR as a kind of human reasoning at an atomic level or fundamental level. 
This article mainly is motivated by (Sun \& Finnie, 2007), in which the authors mainly provided treatment of EBR in the setting of fuzzy logic [37] [10]. However, fuzzy logic and fuzzy reasoning is an extension of traditional mathematical logic and reasoning. In this way, a logical approach to EBR becomes significant in order to develop EBR with applications. Therefore, this article can be considered as the further treatment of EBR in logical setting based on (Sun \& Finnie, 2007).

Furthermore, CBR is a kind of EBR [36]. A logical relationship between CBR and EBR has been examined in [3]. The consideration through Fig. 2 reveals the further relationships between EBR and CBR through eight different CBR reasoning paradigms.

In the context of knowledge base systems (KBS) [11], the introduction of the proposed inference rules for EBR might lead to knowledge inconsistency in the knowledge base and inference inconsistency. For example if we have $P$ and $P \rightarrow Q$ we can deduce $Q$ and $\neg Q$ based on modus ponens or modus ponens with trick. This leads to an inconsistent situation. The same is true for other mentioned inference rules and the corresponding rules with trick. This issue has been mitigated by introducing an agent to analyze the inconsistency of the inferred results [33]. However, how to manage this kind of inconsistency is still a big issue to be solved in future work, just as knowledge inconsistency is still a topic for knowledge base systems and big data with the further development of the Internet.

Minsky [15] argues that we generally like to talk about learning from success rather than from failure, and states that it may be important that we learn from how we fail, learning from failure also leads to more productive thoughts, but in a less directed way. Similarly, in AI, we prefer to search for "islands of consistency" within which ordinary reasoning seems safe [15] and try to avoid knowledge inconsistency and deception. Minsky argues that there are no simple, foolproof ways to get around the inconsistencies of common sense. Common sense reasoning, human reasoning, and EBR usually result from an environment with knowledge inconsistency and inference inconsistency. For example, we can consider all the information on the WWW as a knowledge base. This is the biggest semi-structured knowledge base. It certainly is inconsistent. However, many people usually make decisions based on such a knowledge base or big data, which has become one of the important research frontiers [38]. Therefore how to make decisions in such an environment is certainly a big issue for AI and big data [39]. 


\section{Concluding Remarks}

This article provided a unified logical treatment of experience-based reasoning (EBR). More specifically, it first examined EBR in a general setting. Then it examined eight inference rules for EBR, which cover all possible EBRs from a logical viewpoint, at an atomic level from a Boolean structure viewpoint. Four of them have been thoroughly used in computer science, mathematics, mathematical logic, philosophy and other sciences. The rest have not drawn significant attention in the mentioned areas. However, they are all the abstraction and summary of experience or EBR and human reasoning in real world problems. These eight inference rules constitute the fundamentals for all kind of EBR paradigms. Finally it argued CBR as a kind of EBR through Fig 2. The proposed approach in this research will facilitate the understanding of EBR, $\mathrm{CBR}$, and human reasoning, and their further development with applications to knowledge management and experience management.

In future work we will develop reasoning network based on our EBR reasoning paradigms, and investigate the secrets behind deception and cheat of EBR and human reasoning from a logical viewpoint.

\section{References}

[1] S. Chen, K. H. Tin and A. e. a. Vyas, "A Multi-Layer Dynamic Model for Customer Experience Analytics," Bell Labs Technical Journal, vol. 18, no. 4, pp. 19-32, 2014.

[2] Z. Sun, G. Finnie and J. Sun, "Four new inference rules for experience-based reasoning ," in IFSA2005, Beijing, July 28-31 in Liu Y, Chen G, Ying M (eds) Fuzzy Logic, Soft Computing and Computational Intelligence (IFSA2005), Beijing, 2005.

[3] Z. Sun and G. Finnie, Intelligent Techniques in E-Commerce: A Case-based Reasoning Perspective, Heidelberg, Berlin: Springer-Verlag, 2004, 2010.

[4] E. Stroulia and A. K. Goel, "Generic teleological mechanisms and their use in case adaptation," in 14th Conf Cognitive Science Society, Bloominton, Indiana, July 29-August 1, 1992.

[5] R. Bergmann and K. D. Althoff, "Methodology for building CBR applications," in Case-Based Reasoning Technology, from Foundations to Applications, Berlin, Springer, 1998, pp. 299-326.

[6] Z. Sun and G. Finnie, “A fuzzy logic approach to experience based reasoning," International Journal of Intelligent Systems, vol. 22, pp. 867-889, 2007.

[7] P. N. Johnson-Laird, S. S. Khemlani and . G. P. Goodwin, "Logic, probability, and human reasoning," 
Trends in Cognitive Sciences, vol. 19, no. 4, pp. 201-214, 2015.

[8] D. B. Leake, "Focusing construction and selection of abductive hypotheses," in Proc 11th Int Joint Conf on Artificial Intelligence, 1993, pp. 24-29.

[9] Z. Sun, G. Finnie and K. Weber, “Abductive Case based Reasoning," International Journal Intelligent Systems, vol. 20(9), pp. 957-983, 2004.

[10] H. J. Zimmermann, Fuzzy Set Theory and its Applications (4 ${ }^{\text {th }}$ Edition), Boston/Dordrecht/London: Kluwer Academic Publishers, 2001.

[11] S. Russell and P. Norvig, Artificial Intelligence: A Modern Approach (3rd Edition), Upper Saddle River, NJ: Prentice Hall, 2010.

[12] S. S. Epp, Discrete Mathematics with Applications, Brooks/Cole Publishing Company Pacific Grove, 1995.

[13] P. Torasso, L. Console, L. Portinale and D. Theseider, "On the role of abduction," ACM Computing Surveys, vol. 27 (3), pp. 353-355, 1995.

[14] Z. Sun and G. Finnie, "Brain-like Architecture and Experience-based Reasoning," in Proc. 7th Joint Conf on Information Sciences (JCIS), September 26-30,, Cary, North Carolina, USA, 2003.

[15] M. Minsky, The society of mind, London: Heinemann, 1985.

[16] N. J. Nilsson, Artificial Intelligence: A New Synthesis., San Francisco, California: Morgan Kaufmann Publishers, Inc, 1998.

[17] S. Reeves and M. Clarke, Logic for computer science., Wokingham, England: Addison-Wesley Publishing, 1990.

[18] Wikipedia, "Rules of inference," 2015. [Online]. Available:

http://en.wikipedia.org/wiki/Rules_of_inference.

[19] M. E. Szabo, The Collected Articles on Gerhard Gentzen, Amsterdam: North-Holland Publishing Company, 1969.

[20] C. Baral, “Abductive reasoning through filtering,” Artificial Intelligence, vol. 120, pp. 1-28, 2000.

[21] L. Console, D. Theseider Dupre and P. Torasso, "On the relationship between abduction and deduction," J. Logic Comput, vol. 1, no. 5 , pp. 661-690, 1991.

[22] E. Rich and K. Knight, Artificial Intelligence (2rd edn.), New York: McGraw-Hill, 1991.

[23] H. Kindler, D. Densow, B. Fischer and T. Fliedner, "Mapping laboratory medicine onto the select and test model to facilitate knowledge-based report generation in laboratory medicine," in LNAI 934, Berlin, Springer, 1995.

[24] L. Magnani, Abduction, Reason, and Science, Processes of Discovery and Explanation, New York: Kluwer Academic/Plenum Publishers, 2001.

[25] A. Ciampolini, E. Lamma, P. Mello, Stefanelli and C, "Abductive coordination for logic agents," in ACM Symposium on Applied Computing (SAC'99), San Antonio, Texas, 1999. 
[26] Z. Sun and K. Weber, "Turing test and intelligence with trick," in Proc 8th Ireland Conf on AI (AI-97), Londonderry, Ireland, 1997.

[27] A. M. Turing, “Computing machinery and intelligence,” Mind, vol. 54 (236) , pp. 433-460, 1950.

[28] J. Hutchens, "How to pass the Turing test by cheating," TR 97-05, The Centre for Int. Inf. Proc. Sys., The Univ. of Western Australia, 1997.

[29] M. L. Mauldin, "Chatterbots, Tinymuds, And the Turing Test: Entering the Loebner prize Competition," in Proc. AAAI-94, USA, August, 1994.

[30] T. Sun, The Art of War, China: Guangxi Nationality Press, 1995.

[31] Z. Sun and K. Weber, "Logic with Trick," in Advances in Artificial Intelligence and Engineering Cybernetics, Vol. IV: Systems Logic \& Neural Networks, The Int Inst for Advanced Studies in Systems Research and Cybernetics, 1998, pp. 44-49.

[32] G. Weiss, Multiagent Systems: A modern approach to distributed artificial intelligence, Cambridge, Massachusetts London, England: The MIT Press, 1999.

[33] Z. Sun and G. Finnie, "MEBRS: A Multiagent Architecture for an Experience Based Reasoning System," in Khosla R, Howlett RJ, Jain LC (eds) LNAI 3681, Knowledge-Based Intelligent Information and Engineering Systems: 9th Intl Conf KES2005, Melbourne, September 14, Berlin Heidelberg, 2005.

[34] D. Zak, Programming with Microsof Visual Basic®. NET, THOMSON Course Technology, 2002.

[35] M. Lenz, B. Bartsch-Spörl, H. D. Burkhard and S. Wess, Case-Based Reasoning Technology, from Foundations to Applications, Berlin: Springer, 1998.

[36] G. Finnie and Z. Sun, “A logical foundation for the CBR Cycle,” Int J Intell Syst, vol. 18, no. 4, pp. $367-382,2003$

[37] D. Dubois, F. Esteva, P. Garcia and L. Godo, "Case-based reasoning: A fuzzy approach,” in Ralescu, A.L., Shanahan, J.G. (eds) Fuzzy Logic in Artificial Intelligence, IJCAI’97 Workshop, Berlin, 1999.

[38] C. P. Chen and C.-Y. Zhang, "Data-intensive applications, challenges, techniques and technologies: A survey on Big Data,” Information Sciences, vol. 275 , p. 314-347, 2014.

[39] V. Mayer-Schönberger and K. Cukier, Big Data: A Revolution That Will Transform How We Live, Work, and Think, Eamon Dolan/Houghton Mifflin Harcourt, 2013. 